\title{
Tuberculosis Affecting Multiple Vertebral Bodies
}

\author{
Hideo Baba, Atsushi Tagami, Shinji Adachi, Takeshi Hiura, Makoto Osaki \\ Department of Orthopaedic Surgery, Graduate School of Medicine, Nagasaki University, Nagasaki, Japan
}

Spinal tuberculosis usually occurs in a single vertebral body or two to three adjacent vertebrae; it rarely occurs in multiple vertebral bodies. Surgery is indicated in cases that do not improve with conservative therapy, or when paralysis is evident. Two cases regarding patients with spinal tuberculosis in multiple vertebral bodies on whom surgery was performed are reported. Case 1, the patient was a 77-year-old woman with spinal tuberculosis in four vertebral bodies from the lower thoracic to the lumbar spine. As she had pronounced lower back pain, posterolateral fusion with a pedicle screw was performed. Case 2, the patient was a 29-year-old Indonesian man with spinal tuberculosis in 17 vertebral bodies of the spine who was unable to stand due to paralysis of both legs, thus posterolateral fusion with a pedicle screw was performed. Good results were obtained from tuberculostatic drug therapy and surgical instrumentation.

Keywords: Spinal tuberculosis; Instrumentation; Antitubercular agents

\section{Introduction}

The number of tuberculosis patients in Japan declined between 1950 and 1997, but there has been no clear decrease since 1997, as it remains a common disease. Spinal tuberculosis is also occasionally encountered, but in most cases, it improves with conservative therapy using tuberculostatic agents in order to treat pulmonary tuberculosis. Surgery is indicated, however, in cases that do not improve with conservative therapy, or when paralysis is evident. In general, spinal tuberculosis usually occurs in a single vertebral body or two to three adjacent vertebrae; it rarely occurs in multiple vertebral bodies $[1,2]$. The treatment of two patients with spinal tuberculosis in multiple vertebral bodies, on whom surgery was performed, is reported.

\section{Case Reports}

\section{Case 1}

The patient was a 77-year-old woman. Other than oral medication for hypertension, her medical history was unremarkable. Lower back pain had begun 11 months prior. This gradually worsened, and fever also appeared. Nine months before she was referred to our department, she was admitted to a general hospital because of difficulty sitting. A fever ranging from $37.5^{\circ} \mathrm{C}$ to under $39^{\circ} \mathrm{C}$ was observed. Laboratory data showed no elevation of white blood cell (WBC, 3600/ $\mu \mathrm{L}$ ), but C-reactive protein (CRP) levels were elevated $(3.33 \mathrm{mg} / \mathrm{dL})$. Both miliary tuberculosis and multiple bone tuberculosis at levels Th7, 12, L3, 4,5 , the ribs, right ilium, and left ischium were evident. Tuberculosis drug therapy was initiated with four agents. Her fever subsided as the miliary tuberculosis improved,

Received Feb 15, 2012; Revised May 14, 2012; Accepted Jun 2, 2012

Corresponding author: Makoto Osaki

Department of Orthopaedic Surgery, Nagasaki University Graduate School of Medical Science,

1-7-1 Sakamoto, Nagasaki 8528501, Japan

Tel: +81-95-819-7321, Fax: +81-95-849-7325, E-mail: mosaki@nagasaki-u.ac.jp 
but the lower back pain persisted, and she was referred to our department due to experiencing difficulty in sitting and standing.

There was pronounced tenderness in the lumbar vertebrae despite the absence of neurological abnormalities. Radiographic imaging showed osteolytic changes to the vertebral bodies of the lumbar spine, and multiple osteolytic changes at levels Th12, L3, L4, and L5 were evident on computed tomography (CT), which were particularly pronounced in the L4 vertebral body (Fig. 1). Magnetic resonance imaging (MRI) also showed the same inflammatory granulation or abscesses in Th12, L3, L4, and L5 observed on CT (Fig. 2). On the pathological examination of a bone biopsy, Langhans giant cells, caseous necrosis, and acid-fast bacteria were seen, and a diagnosis of spinal tuberculosis was made.

Surgery was performed because the patient had difficulty sitting due to lower back pain. The surgery involved posterolateral fusion with pedicle screws from L2 to S1 (Fig. 3). Compared to L3, 4, and 5, the vertebral osteolysis in Th12 was mild and not the cause of the lower back pain. Furthermore, as the osteolysis was mild, the probability of vertebral fracture occurring in the future was judged to be low, and, therefore, Th12 was not fused. The patient's postoperative course was uneventful, and her lower back pain improved. As of 18 months postoperatively, the patient was ambulatory.

\section{Case 2}

The patient was a 29 -year-old Indonesian man. His pre-

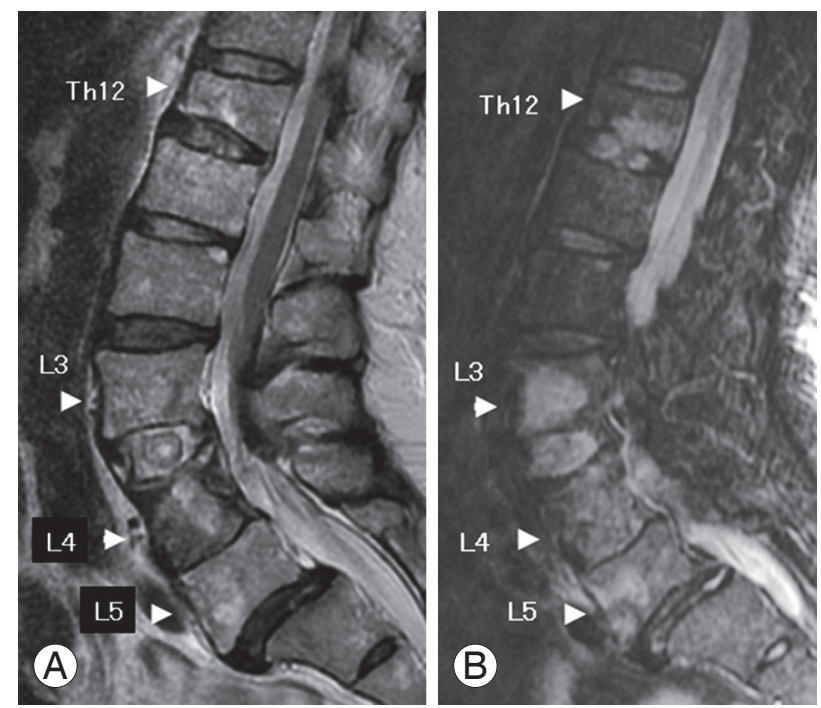

Fig. 2. Sagittal T2-weighted magnetic resonance images (A) and parasagittal T2 weighted short tau inversion recovery images (B) show the inflammatory granulation or abscesses in Th12, L3, L4, and L5 (arrowheads).
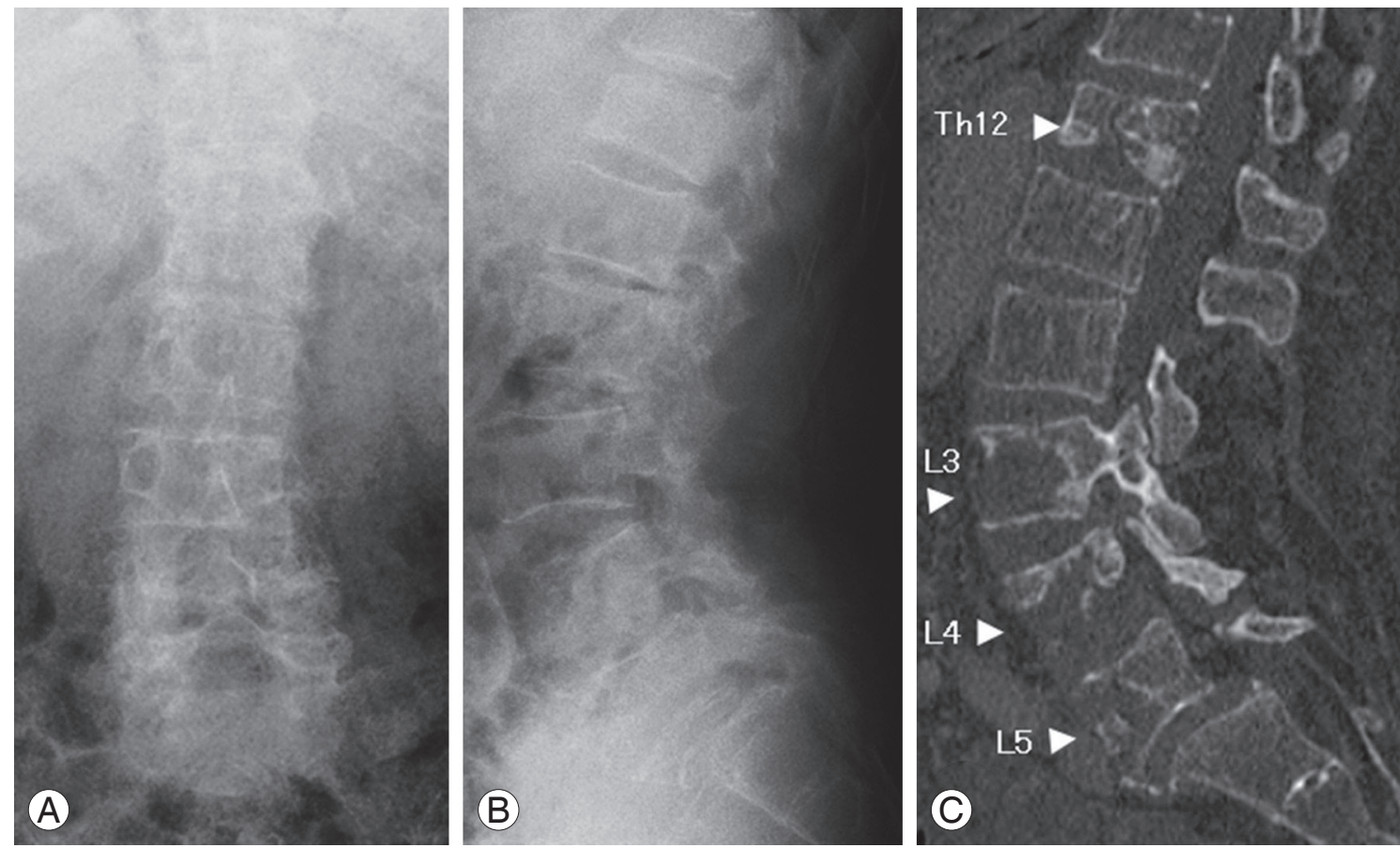

Fig. 1. Case 1. Anteroposterior (A) and lateral (B) radiography of the lumbar spine of a 77-year-old woman. (C) Computed tomography imaging shows multiple osteolytic changes at Th12, L3, L4, and L5 (arrowheads), which are particularly pronounced in the L4 vertebral body. 
vious medical history was unremarkable. He began to experience back pain 14 months earlier. This gradually worsened, and since fever also appeared, he was admitted to a general hospital 6 months before he was referred to our department. The patient had a persistent fever, ranging from $39^{\circ} \mathrm{C}$ to under $41^{\circ} \mathrm{C}$. Laboratory data showed no elevation of WBC (WBC, $8200 / \mu \mathrm{L})$, but inflammatory findings were seen (CRP $12.2 \mathrm{mg} / \mathrm{dL}$; erythrocyte sedimentation rate [ESR], 128/142 mm). He was diagnosed with multiple bone tuberculosis. Furthermore, the patient tested negative on the anti-HIV antibody test and did not have AIDS. Tuberculosis drug therapy was initiated with four agents. Since testing showed that the organism exhibited good sensitivity to the four types of tuberculostatic agents, and the abscesses improved markedly following the start of treatment, the patient did not have multi-drug resistant tuberculosis. The back pain and fever gradually improved, but from 5 months prior to the referral, reduced sensation in both legs and a sense of weakness appeared, and the patient became incapable of walking unaided. His symptoms subsequently progressed further, and he was examined in our department.

Loss of muscular strength was pronounced, particularly in the right leg, and the patient exhibited difficulty standing. Hypesthesia below Th10 and urinary and defecatory disturbances were evident. The tendon reflex was enhanced in all four limbs, and the Babinski reflex and ankle clonus were both present bilaterally. Radiographic
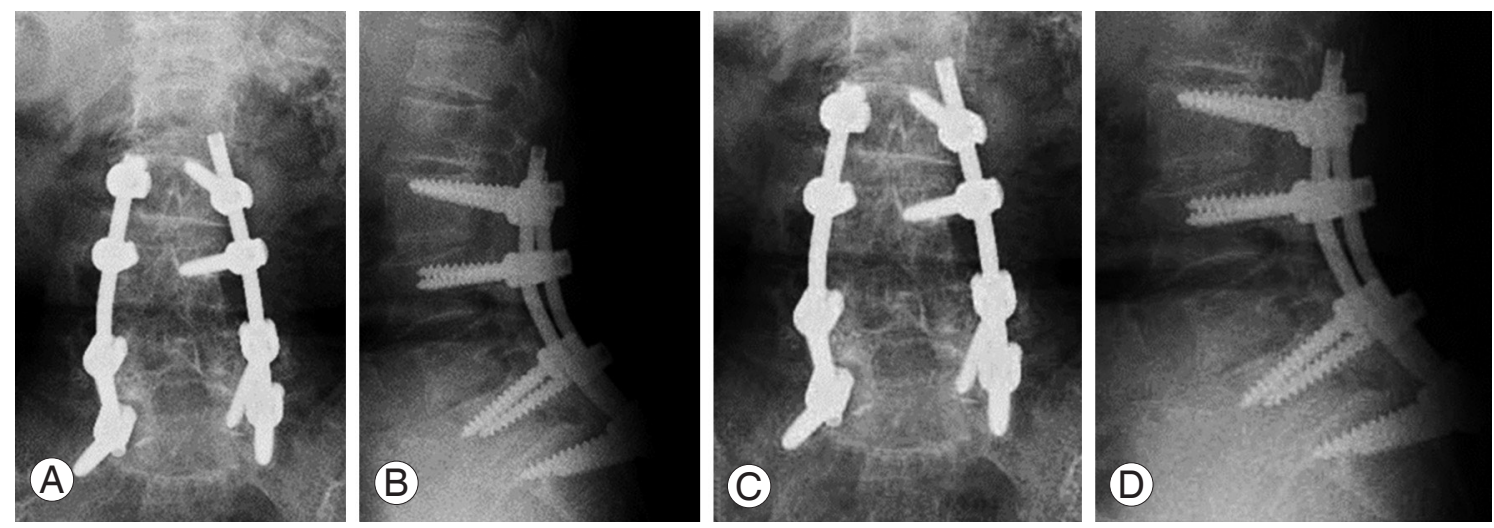

Fig. 3. (A, B) The surgery involved posterolateral fusion with pedicle screws from L2 to S1. (C, D) As of 18 months postoperatively, the patient was ambulatory.
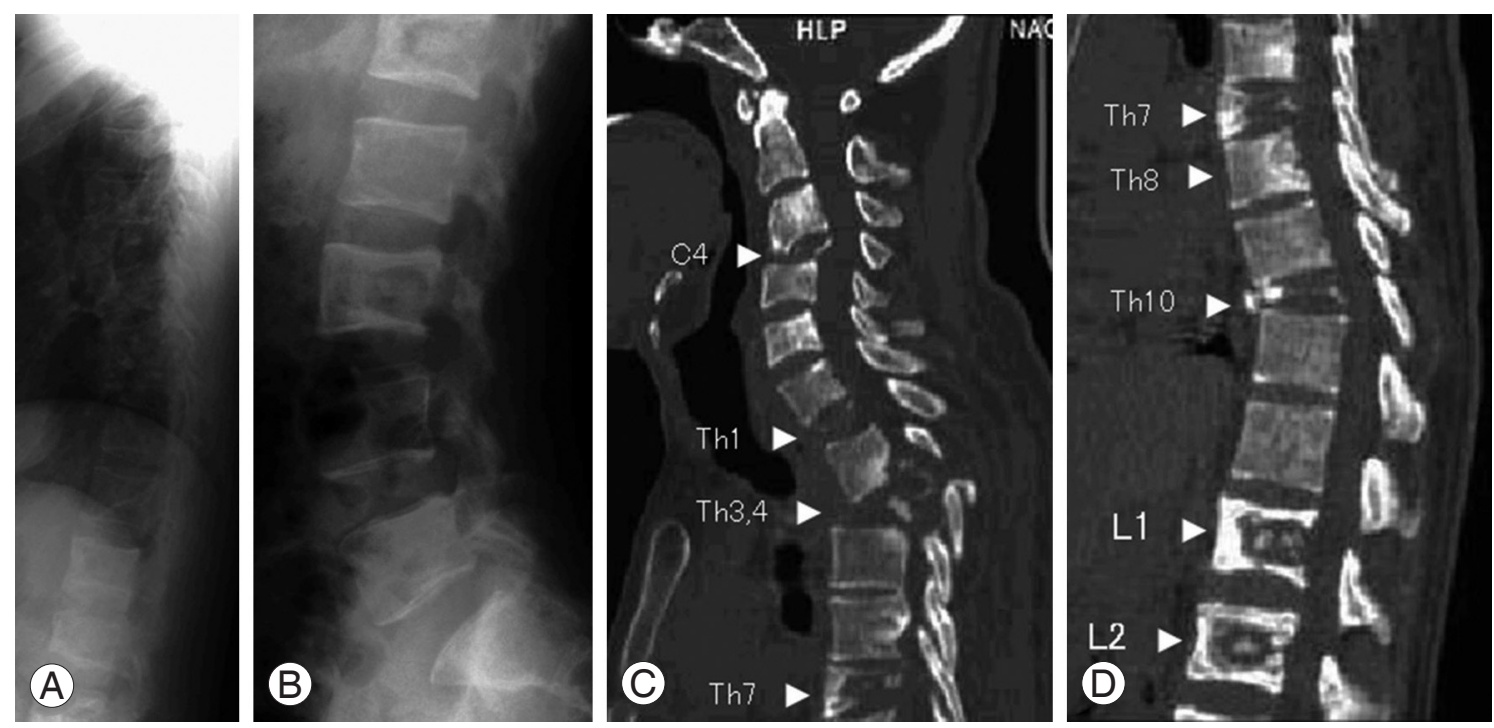

Fig. 4. Case 2. Lateral radiography of the thoracic (A) and lumbar (B) spine of a 29-year-old Indonesian man. Radiographic imaging showed widespread osteolytic lesions in the vertebral bodies of the thoracolumbar spine. (C, D) Computed tomography imaging shows 17 affected vertebral bodies: C3, C4, C6, C7, Th1-8, Th10, L1-3, and L5. Multiple osteolytic changes are evident in C4, C6, Th1, Th3, Th4, Th7, Th8, Th10, L1, and L2, and they are particularly marked in C4, Th1, Th3, Th4, Th7, Th10, and L1 (arrowheads). 
imaging showed widespread osteolytic lesions in the vertebral bodies of the thoracolumbar spine. The CT showed 17 affected vertebral bodies: C3, C4, C6, C7, Th1-8, Th10, L1-3, and L5. Multiple osteolytic changes were evident in C4, C6, Th1, Th3, Th4, Th7, Th8, Th10, L1, and L2, and they were particularly marked in C4, Th1, Th3, Th4, Th7, Th10, and L1 (Fig. 4). MRI also demonstrated the same destruction of multiple vertebral bodies evident on the $\mathrm{CT}$, with compression of the spinal cord by inflamma-

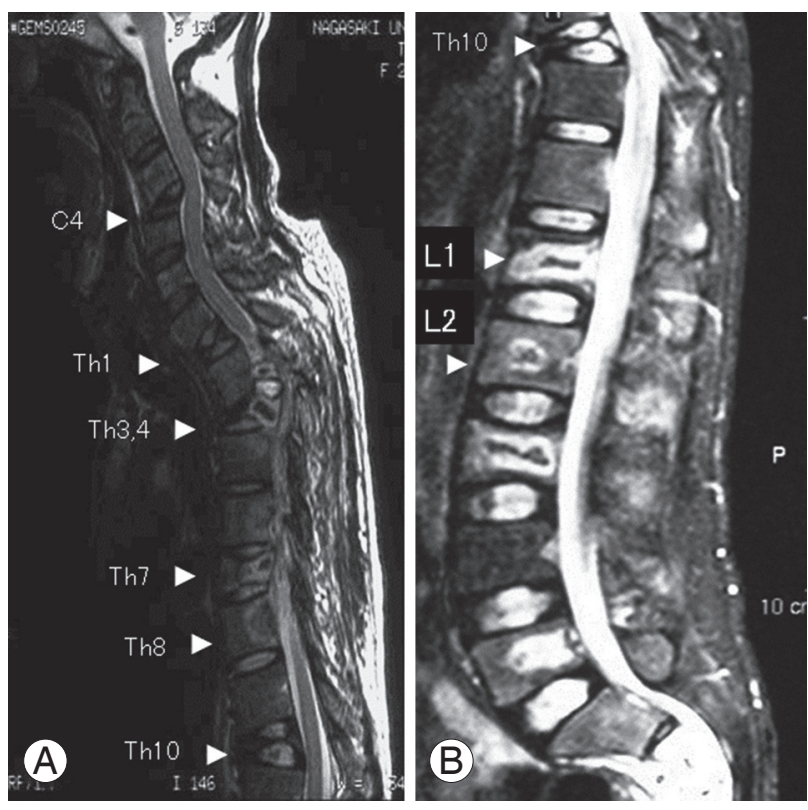

Fig. 5. (A, B) Sagittal T2-weighted images show the destruction of multiple vertebral bodies evident on the computed tomography (arrowheads), with compression of the spinal cord by inflammatory granulation and abscesses at Th1/2 and Th3/4. tory granulation and abscesses at Th $1 / 2$ and Th $3 / 4$ (Fig. 5). Pathological findings from bone biopsy examination showed Langhans giant cells, caseous necrosis, and acidfast bacteria, and a diagnosis of spinal tuberculosis was made.

Surgery was performed two months after he was referred to our department due to the paralysis of both legs. Inflammatory granulation and abscesses were seen primarily in the spinal canal and were not observed in the vertebral bodies. We therefore judged that the probability of vertebral osteolysis progressing in the future was low, and that anterior reconstruction was not necessary. We also judged that long fusion would enable the prevention of sequential collapse, and that the posterior single approach was indicated. Therefore, the surgery involved posterolateral fusion with pedicle screws from C6 to Th6 after laminectomy (Fig. 6). The patient's postoperative course was uneventful, and his paralysis improved. Four months after surgery, he was capable of independent gait, and the urinary and defecatory disturbance had also improved. Since the tuberculous spondylitis had resolved one year after use of tuberculostatic agents, treatment regarding the use of these agents was discontinued.

\section{Discussion}

According to a report by the Research Institute of Tuberculosis of the Japan Antituberculosis Association, the number of tuberculosis patients in Japan remains high, at around 23,000 cases per year, showing no tendency
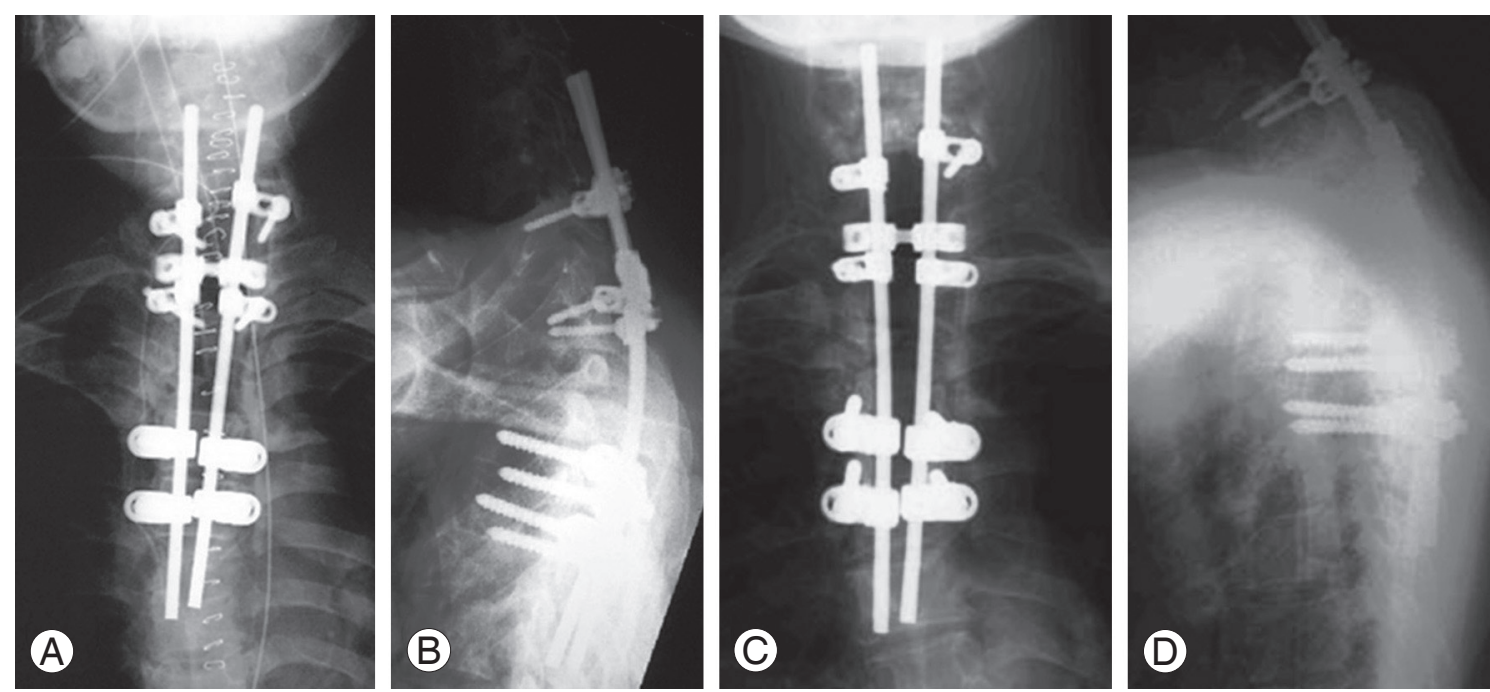

Fig. 6. (A, B) The surgery involves posterolateral fusion with pedicle screws from C6 to Th6 after laminectomy. (C, D) As of four months postoperatively, the patient was capable of independent gait, and the urinary and defecatory disturbances also improved. 
of decline. The situation is the same for bone tuberculosis, with approximately 500 patients per year. Japan has a high number of tuberculosis patients compared with developed countries in Europe and North America, with a prevalence 4.5 times that of the United States. In Southeast Asia, however, the incidence is still higher, and in Indonesia, the homeland of case 2, the prevalence is 6.5 times that of Japan. With an increasing number of visitors to Japan from overseas, the proportion of tuberculosis patients in their 20s in Japan who are of foreign nationality now stands at $30 \%$. Thus, there is a high probability for an increase in the number of tuberculosis patients within Japan in the future, and attention is already required in routine medical practice.

Bone tuberculosis is usually localized, and its multiple occurrence is rare $[3,4]$. Spinal tuberculosis is the most frequently occurring form of bone tuberculosis, but in most cases it occurs in a single vertebral body or two to three adjacent vertebrae [1,2]. Zhang and Jin [5] reported the case of a 43-year-old woman with multiple bone tuberculosis in the skeleton, and there have been a few other reports regarding multiple skeletal occurrences [24,6-9]. Neher et al. [1] reported a case of multiple spinal tuberculosis in 12 vertebral bodies, but such cases of multiple vertebral bodies being affected are exceptionally rare, and there has been no other report similar to the present case 2 with 17 affected vertebral bodies: C3, C4, C6, C7, Th1-8, Th10, L1-3, and L5. In general, multiple bone tuberculosis is believed to occur in patients with compromised hosts due to complicating conditions, steroid therapy, or other reasons [5]. In the cases reported here, however, the cause of multiple bone tuberculosis was unclear, since case 1 only had a history of hypertension, and case 2 was a 29-year-old individual with no previous medical history. It must be noted that multiple bone tuberculosis may occur even in patients with no history of a complicating condition.

Case 1 underwent posterolateral fusion of multiple levels with pedicle screws because her lower back pain did not improve despite extensive conservative treatment. Case 2 underwent laminectomy and posterolateral fusion of multiple levels with pedicle screws using a posterior approach due to paralysis of both legs. In both cases, the postoperative course was uneventful. This indicates that even patients with spinal tuberculosis affecting mul- tiple vertebral bodies can be treated proactively through the posterolateral fusion of multiple levels with pedicle screws using a posterior approach. Good results were obtained from tuberculostatic drug therapy and surgical instrumentation.

\section{Conflict of Interest}

No potential conflict of interest relevant to this article was reported.

\section{References}

1. Neher A, Kopp W, Berna G, Frank J, Kohlhaufl M. Advanced multifocal tuberculous spondylitis without disk involvement and with multidrug-resistant bacilli. Clin Infect Dis 2007;45:e109-12.

2. Hong L, Wu JG, Ding JG, et al. Multifocal skeletal tuberculosis: experience in diagnosis and treatment. Med Mal Infect 2010;40:6-11.

3. Tiwari A, Sud A, Mehta S, Kanojia RK, Kapoor SK. Multifocal skeletal tuberculosis presenting as multiple bone cysts. Ann Acad Med Singapore 2007;36:10389.

4. Gelal F, Sabah D, Dogan R, Avci A. Multifocal skeletal tuberculosis involving the lumbar spine and a sacroiliac joint: MR imaging findings. Diagn Interv Radiol 2006;12:139-41.

5. Zhang H, Jin D. Multiple cystic tuberculous lesions in the skeleton (Jungling disease): a case report. Spine (Phila Pa 1976) 2003;28:E155-7.

6. Morris BS, Varma R, Garg A, Awasthi M, Maheshwari M. Multifocal musculoskeletal tuberculosis in children: appearances on computed tomography. Skeletal Radiol 2002;31:1-8.

7. Marudanayagam A, Gnanadoss JJ. Multifocal skeletal tuberculosis: a report of three cases. Iowa Orthop J 2006;26:151-3.

8. Sezgin B, Atilganoglu U, Yigit O, Ergun SS, Cambaz $\mathrm{N}$, Demirkesen C. Concomitant cutaneous metastatic tuberculous abscesses and multifocal skeletal tuberculosis. Indian J Dermatol 2008;53:149-53.

9. Rahman MA, Mutanabbi M, Rahman MH, Arefin KE, Helal MA. Multifocal skeletal tuberculosis in children. Mymensingh Med J 2009;18:109-12. 